\title{
Mass cytometry reveals an impairment of B cell homeostasis in Anti-synthetase syndrome
}

\section{Gaëlle Dzangué-Tchoupou ${ }^{1 *}$, Yves Allenbach ${ }^{1,2}$, Corinna Preuße $^{3}$, Werner Stenzel $^{3}$, Olivier} Benveniste $^{1,2}$

${ }^{1}$ Centre of research in Myology, Sorbonne Université, INSERM, Association Institut de Myologie, UMRS 974, 75013, Paris, France.

${ }^{2}$ Department of Internal medicine and clinical immunology, Pitié-Salpêtrière University hospital, DHU I2B, APHP, INSERM, UMR 974, 75103, Paris, France.

${ }^{3}$ Department of Neuropathology, Charité - Universitätsmedizin Berlin, 10117, Berlin, Germany.

*Corresponding author: Gaëlle Dzangué-Tchoupou

Mail: gaelle.dzangue@gmail.com

105 Boulevard de l'hôpital, $3^{\text {rd }}$ floor, 75013, Paris-France.

Phone number: 00 (33) $0140779684 \quad$ Fax: 00 (33) 0140778129

Declarations of interest: none. 


\section{Abstract:}

Recent data suggest the implication of T, B and NK cells in the anti-synthetase syndrome (ASyS); nevertheless their role and activation states are poorly described.

We performed deep immune-profiling using 37 markers on peripheral blood cells from 10 ASyS patients versus 17 healthy donors (HD) and 26 myositis control patients.

We show decreased percentages of memory B cells in ASyS patients (mean \pm SEM: ASyS $=13 \pm 3 \%, H D=37 \pm 4 \%$ and 'myositis controls' $=32 \pm 3$ ), counterbalanced by increased percentages of naïve B cells. Interestingly, perifascicular infiltrations of memory B cells within muscle biopsies of ASyS patients suggest that they niche within the muscle.

Key words: anti-synthetase syndrome, myositis, B cell homeostasis, deep immune profiling, mass cytometry 


\section{Introduction:}

The anti-synthetase syndrome (ASyS) is an autoimmune disorder characterized by the presence of myositis, interstitial lung disease, Raynaud's phenomenon, arthritis and antibodies directed against amino-acyl-t-RNA synthetases (Benveniste et al., 2016; Marguerie et al., 1990) The progressive pulmonary disease observed in ASyS patients leads to poor prognosis. Approximately eighty percent of ASyS patients present antibodies directed against the histidyl t-RNA synthetase (Jo1) (Benveniste et al., 2007).

In patients presenting anti-Jo1 antibodies, antibody titers have been associated with disease activity (Stone et al.). Immunization of mice with purified murine Jo1 peptides leads to the generation of activated $T$ and $B$ cells infiltrating the muscles and lungs, and directed against species-specific Jo1 epitopes (Katsumata et al., 2007). More recently, our group observed a differentiated NK cell profile in active ASyS anti-Jo1 patients (CD57+ NK cells), accompanied by substantial infiltrations of NK cells within the lungs of patients compared to healthy donors (Hervier et al., 2016). Altogether, these studies suggest the pathogenic role of Jo1 and anti-Jo1 antibodies, and the implication of B, T and NK cells in the physiopathology of anti-Jo1 ASyS.

Cytometry by time-of-flight (CyTOF) is a powerful single-cell immune profiling tool, which allows the simultaneous analysis of more than 37 markers using metal-conjugated antibodies (Dzangué-Tchoupou et al., 2019). This tool is of particular interest in the discovery of novel cell-populations and biomarker identification. Associated with barcoding using palladium isotopes, CyTOF allows the identification of subtle differences within populations and different experimental groups (Dzangué-Tchoupou et al., 2018).

The aim of this study was to identify specific cell populations potentially involved in the physiopathology of anti-Jo1 ASyS.

\section{Material and methods:}

2.1. Ethical approval: Approval for this study was obtained from the Comite Consultatif sur le Traitement de l'Information en matière de la Recherche dans le domaine de la Santé (CCTIRS) France ( $\left.\mathrm{N}^{\circ} 14.323\right)$ and all patients and healthy controls signed a written informed consent. 
2.2. Human samples: In an attempt to identify populations, which could be implicated in the physiopathology of anti-Jo1 ASyS $(n=10)$, we screened their peripheral blood mononuclear cells (PBMC) using 36 markers by CyTOF barcoding. Firstly, mass cytometric data from ASyS patients was compared to those of 10 healthy donors. Next, populations of interest were characterized in comparison to a pool of 26 active myositis 'control' patients (MC) comprised of well-defined groups of myositis patients (inclusion body myositis (IBM, $\mathrm{n}=10$ ), anti-3-hydroxy-3-methylglutaryl coA reductase myopathy (HMGCR, $n=9$ ) and antisignal recognition particle myopathy (SRP, $n=7)$ and a total number of 17 healthy controls (HC). Anti-Jo1 patients and myositis control patients were diagnosed according to internationally accepted criteria (Allenbach et al., 2018; Lloyd et al., 2014). All these myositis patients were active and mostly untreated Table 1.

2.3. Mass cytometric experiments: Barcoding, mass cytometric staining, data acquisition and data pre-processing (gating out of beads and dead cells) were performed as previously described in a technical research article (Dzangué-Tchoupou et al., 2018). The list of antibodies used is available in Supplementary Table 1.

\subsection{Data analysis and statistics:}

2.4.1. Using the 'Citrus' algorithm available in the Cytobank cloud-based platform, we performed automated identification of stratifying signatures in cellular populations. Thus, in order to identify main populations of interest, 'Citrus' was applied on 10 anti-Jo1 ASyS patients vs. 10 healthy donors.

2.4.2. The next step was to characterize populations of interest between anti-Jo1 ASyS patients, $\mathrm{HC}$ and $\mathrm{MC}$ in detail. For that, main cell populations such as $\mathrm{B}, \mathrm{T}$ and NK cells were exported automatically using automated cell clustering using the "spanning-progression tree of density-normalized events" (SPADE) algorithm (available in the Cytobank cloud-based platform) for downstream analysis.

2.4.3. Data was further analyzed using supervised approaches such as dot plots and histograms. Statistics was performed using Graphpad prism software version 6. We used Shapiro-Wilk normality test to verify the distribution of quantitative data among the groups compared. In-text results are represented as mean \pm SEM.

2.5. Immunostainings: Muscle biopsies from two anti-Jo1 ASyS were stained using antiCD27 (cyanine 3; red), anti-CD20 (Alexafluor 488; green) and 4',6-diamidino-2-phenylindole (DAPI; blue) to stain the nuclei. 


\section{Results:}

Mean ages of anti-Jo1 patients, $\mathrm{HC}$ and $\mathrm{MC}$ were respectively, $48 \pm 4,52 \pm 3$ and $52 \pm 2$ years (Table 1). The Citrus algorithm identified an increase in the expressions of CD57 within NK cells (CD56+ CD16+), IgD within B cells (CD19+) and CD38 within CD8 T cells (CD3+ CD8+) in ASyS patients $(n=10)$ compared to healthy donors $(n=10)$ (Supplementary Figure 1).

Next, we aimed at characterizing these cell populations between ASyS patients $(n=10), H C(n=17)$ and $M C(n=26)$ in detail. For that, NK, B and CD8+ T cells were exported from automated SPADE trees for downstream analysis. Concerning NK cells, we show an increase in differentiated CD57+ NK cells in anti-Jo1 patients: anti-Jo1 $=64 \pm 4 \%$ vs (HC $=$ $49 \pm 3 \%, p=0.07$ and $M C=46 \pm 4 \%, p=0.01$ ) (Supplementary Figure 2). Considering that this observation was previously reported (Hervier et al., 2016), we decided to focus on B cells and CD8+ T cells.

We observed a decrease in the frequency of memory B cells (CD19+ CD27+) in antiJo1 patients: anti-Jo1 $=13 \pm 3 \%$ vs $(H C=37 \pm 4 \%, p<0.0001$ and $M C=32 \pm 3 \%, p=0.001)$, counterbalanced by an increase in the frequency of naive B cells (CD19+ CD27-): anti-Jo1 = $86 \pm 3 \%$ vs ( $H C=61 \pm 4 \%, p<0.0001$ and $M C=66 \pm 3, p=0.001$ ) (Figure 1 ). We did not observe changes in the frequency of CD95+ or HLA-DR+ B cell populations between anti-Jo1 ASyS patients in comparison to control groups (Figure 1).

Concerning CD8+ T cells, the frequency of CD38+ CD8+ T cells was increased only in control myositis patients compared to healthy controls. Nevertheless, activated HLA-DR+ CD8 T cells were increased in all myositis groups compared to healthy controls: $H C=2 \pm 0.3 \%$ vs (anti-Jo1 $=5 \pm 1 \%, p=0.02$ and $M C=4 \pm 0.5, p=0.01$ ).

Finally, immunostainings performed on skeletal muscle biopsies from anti-Jo1 patients revealed the presence of perifascicular infiltrates of CD20+CD27+ memory B cells (Figure 2). This data suggest that memory B cells niche within the muscle in anti-Jo1 ASyS patients. We performed additional immunostains of CD27 and CD20 on patients with dermatomyositis, patients with sIBM, patients with IMNM and with anti-Jo1 Abs (Supplementary Figure 3). Results show that, while skeletal muscle biopsies from patients with IMNM and sIBM lack infiltrating memory B cells (CD20+ CD27+), DM and anti-Jo1 
patients show infiltrating memory B cells. The presence of memory B cells in DM biopsies is in line with the literature (Radke J. et al. 2018).

\section{Discussion:}

Despite the presence of specific auto-antibodies in anti-Jo1 ASyS patients, the role of B cells in the course of the disease is poorly described. Using mass cytometry, we show that within a homogenous group of patients presenting anti-Jo1 antibodies, there is an impairment of the B cell compartment in comparison to control groups (healthy controls and a pool of three other sub-groups of myositis patients). We show that memory B cells are decreased in peripheral blood of anti-Jo1 ASyS patients compared to control groups. Unfortunately, we do not have access to PBMC from untreated patients with other antisynthetase antibodies. In addition, we show the presence of memory B cells within the muscle of anti-Jo1 ASyS patients, which is one of the organs targeted by the disease. The dysregulation of B cell homeostasis in anti-Jo1 patients is unexpected, since neither other myositis patients presenting myositis specific autoantibodies (anti-HMGCR and anti-SRP myopathies) nor IBM patients present this impairment. This observation suggests the possibility that antigen-specific memory $B$ cells home to target organs such as muscle, where they carry out effector functions.

We also observed an increase in the frequency of differentiated CD57+ NK cells in anti-Jo1 ASyS patients compared to control groups, in line with previous published data (Hervier et al., 2016).

In line with recent published data which observed an expansion of CD57+ CD8+ T cells in sIBM patients compared to HC or other myositis patients (Dzangué-Tchoupou et al., 2019; Greenberg et al., 2016), here we show that this cell population increases in the pool of 'myositis control' patients compared to healthy controls, particularly in sIBM patients (supplementary Figure 4). Nevertheless, this CD57+CD8+ T cell population is not increased in ASyS anti-Jo1 patients.

Katsumata et al., (2007) showed that immunization of mice with purified murine Jo1 peptides leads to the generation of activated $T$ and $B$ cells infiltrating the muscles and lungs, and directed against species-specific Jo1 epitopes. In addition, our group (Hervier et al., 2016) observed a differentiated NK cell profile in active ASyS anti-Jo1 patients, accompanied by important infiltrations of NK cells with in the lungs of patients compared to healthy donors. Based on these studies, the implication of $B, T$ and NK cells is very likely in the antisynthetase syndrome. In this study, we show an impairment of B cells homeostasis and confirm the immune NK cell phenotype described previously specifically in antisynthetase syndrome patients and not in other myositis patients. Nevertheless, the presence of activated CD8+ T cells was also observed in SIBM and thus is not specific to 
antisynthetase syndrome myositis. Altogether, amongst 4 groups of myositis patients, we show an impairment of $B$ cell homeostasis and an activated NK cell immune profile specific to ASyS anti-Jo1.

5. Funding: This work was supported by the Association Française contre les myopathies.

\section{References:}

Allenbach, Y., Mammen, A.L., Benveniste, O., Stenzel, W., and Immune-Mediated Necrotizing Myopathies Working Group (2018). 224th ENMC International Workshop:: Clinico-seropathological classification of immune-mediated necrotizing myopathies Zandvoort, The Netherlands, 14-16 October 2016. Neuromuscul. Disord. NMD 28, 87-99.

Benveniste, O., Dubourg, O., and Herson, S. (2007). [New classifications and pathophysiology of the inflammatory myopathies]. Rev. Med. Interne 28,603-612.

Benveniste, O., Stenzel, W., and Allenbach, Y. (2016). Advances in serological diagnostics of inflammatory myopathies. Curr. Opin. Neurol. 29, 662-673.

Dzangué-Tchoupou, G., Corneau, A., Blanc, C., Benveniste, O., and Allenbach, Y. (2018). Analysis of cell surface and intranuclear markers on non-stimulated human PBMC using mass cytometry. PloS One 13, e0194593.

Dzangué-Tchoupou, G., Mariampillai, K., Bolko, L., Amelin, D., Mauhin, W., Corneau, A., Blanc, C., Allenbach, Y., and Benveniste, O. (2019). CD8 + T-bet+ cells as a predominant biomarker for inclusion body myositis. Autoimmun. Rev.

Greenberg, S.A., Pinkus, J.L., Amato, A.A., Kristensen, T., and Dorfman, D.M. (2016).

Association of inclusion body myositis with $\mathrm{T}$ cell large granular lymphocytic leukaemia. Brain J. Neurol. 139, 1348-1360.

Hervier, B., Perez, M., Allenbach, Y., Hervé, D., Cohen, F., Uzunhan, Y., Ouakrim, H., Dorgham, K., Méritet, J.-F., Longchampt, E., et al. (2016). Involvement of NK Cells and NKp30 Pathway in Antisynthetase Syndrome. J. Immunol. Baltim. Md 1950197.

Katsumata, Y., Ridgway, W.M., Oriss, T., Gu, X., Chin, D., Wu, Y., Fertig, N., Oury, T., Vandersteen, D., Clemens, P., et al. (2007). Species-specific immune responses generated by histidyl-tRNA synthetase immunization are associated with muscle and lung inflammation. J. Autoimmun. 29, 174-186.

Lloyd, T.E., Mammen, A.L., Amato, A.A., Weiss, M.D., Needham, M., and Greenberg, S.A. (2014). Evaluation and construction of diagnostic criteria for inclusion body myositis. Neurology 83, 426-433.

Marguerie, C., Bunn, C.C., Beynon, H.L.C., Bernstein, R.M., Hughes, J.M.B., So, A.K., and Walport, M.J. (1990). Polymyositis, Pulmonary Fibrosis and Autoantibodies to AminoacyltRNA Synthetase Enzymes. QJM Int. J. Med. 77, 1019-1038. 
Stone, K.B., Oddis, C.V., Fertig, N., Katsumata, Y., Lucas, M., Vogt, M., Domsic, R., and Ascherman, D.P. Anti-Jo-1 antibody levels correlate with disease activity in idiopathic inflammatory myopathy. Arthritis Rheum. 56, 3125-3131. 
Figure legends:

\section{Figure 1: Impairment of the peripheral B cell compartment in anti-Jo1 patients}

Automated cell clustering was performed using the SPADE algorithm available on cytobank. Next nodes corresponding to B cells were exported for downstream analyses. Here, we describe the percentages of $B$ cell populations amongst patients presenting anti-Jo1 antibodies $(n=13)$, other myositis patients [ $n=26$ : IBM $(n=10)$, anti-SRP myopathy $(n=7)$ and anti-HMGCR myopathy $(n=9)$ ] and age-matched healthy donors $(n=23)$. a) dot plots showing the gating strategy used for naïve and memory $B$ cells, b) comparison of the percentages of naïve and memory $B$ cells between the three groups, c) percentages of CD95+ and HLA-DR+ memory $B$ cells d) percentages of CD95+ and HLA-DR+ naïve B cells and e) immunostainings on muscle biopsies from two ASyS-Jo1+ patients showing memory CD20+ (AF488) / CD27+ (Cy3) cells around muscle fibers.

Statistics was performed with Graphpad Prism version 6. Shapiro-Wilk normality test was used to verify the distribution of quantitative data. Next, we performed comparisons using either one-way ANOVA test with Bonferroni correction or Kruskal-Wallis test with Dunn's correction $\left({ }^{*} p<0.05, * * p<0.001, * * * p<0.0001\right.$ and $\left.* * * * p<0.00001\right)$.

\section{Figure 2: Detection of memory B cells within muscle biopsies of anti-Jo1 patients}

Immunostainings on muscle biopsies from two ASyS-Jo1+ patients showing memory CD20+ (AF488) / CD27+ (Cy3) cells around muscle fibers and DAPI. 
Table 1: clinical characteristics of active ASyS anti-Jo1 patients, active myositis control patients and healthy donors

\begin{tabular}{|l|c|c|c|c|c|}
\hline Disease & SIBM & IMNM anti-HMGCR & IMNM anti-SRP & ASS anti-Jo1 & HD \\
\hline$n=$ & 10 & 9 & 7 & 10 & 17 \\
\hline Age (years) & $59.3 \pm 2$ & $44.1 \pm 6$ & $52.3 \pm 8$ & $47.8 \pm 4$ & $51.5 \pm 3$ \\
\hline Female percentage & 61.1 & 77.7 & 57.1 & 69.2 & na \\
\hline Disease duration (years) & $9 \pm 1$ & $8 \pm 3$ & $3.2 \pm 3$ & $2.5 \pm 1$ & na \\
\hline CK level (IU/L) & $453 \pm 154$ & $3977 \pm 1670$ & $3430 \pm 888$ & $2816 \pm 1141$ & na \\
\hline MMT8 & $132.3 \pm 5$ & $127.9 \pm 8$ & $109.9 \pm 10$ & $134.2 \pm 5$ & na \\
\hline PGA & na & $7.2 \pm 0.4$ & $7.7 \pm 0.4$ & $7.2 \pm 0.5$ & na \\
\hline Treated/untreated patients & $0 / 18$ & $1 * / 8$ & $3 * * / 4$ & $1 \% / 9$ & na \\
\hline Treatment duration ( $\pm S E M)$ & 0 & 4 years & $4 \pm 3$ months & 2 years & na \\
\hline
\end{tabular}

*This patient received CT $(6 \mathrm{mg})$

**For these treated patients, one received CT

(30mg), the other CT (50mg) and the last

IVIG/AZT (150mg)

${ }^{\$}$ This patient received CT ( $\left.5 \mathrm{mg}\right)$ and MTX

(20mg/week)

$\mathrm{CK}=$ creatine phosphokinase

MMT8= manual muscle testing 8

$\mathrm{PGA}=$ physician global assessment

$\mathrm{CT}=$ oral corticosteroid

IVIG= intra veinous Immunoglobulin

$A Z T=$ azathioprine

MTX= methotrexate

na=not applicable 
Highlights

- Recent data suggest the implication of T, B and NK cells in the ASyS

- Detailed phenotypes of physiopathology involved in the subsets are poorly described

- Specific decrease of memory B cells in ASyS associated with increased differentiated NK cells compared to controls

- Presence of memory B cells within muscle biopsies of ASyS, suggesting they home to skeletal muscle

- Impairment of B cell homeostasis in ASyS compared to active myositis patients 
a)

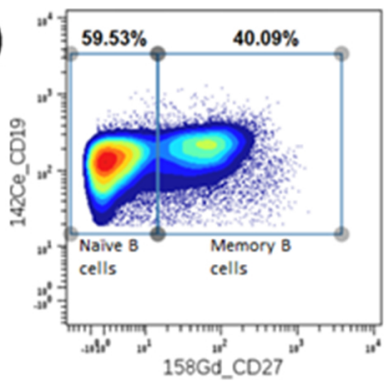

b)

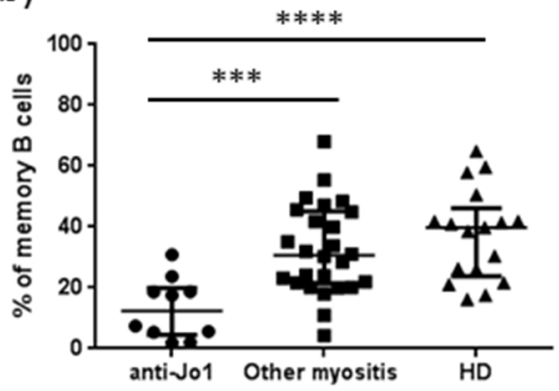

$* * * *$

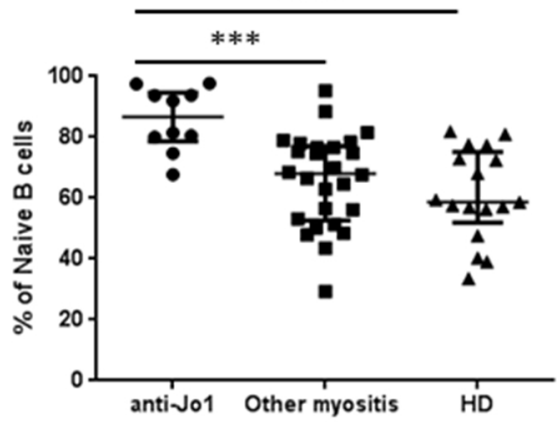

c)

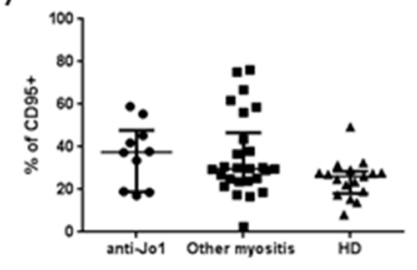

e)

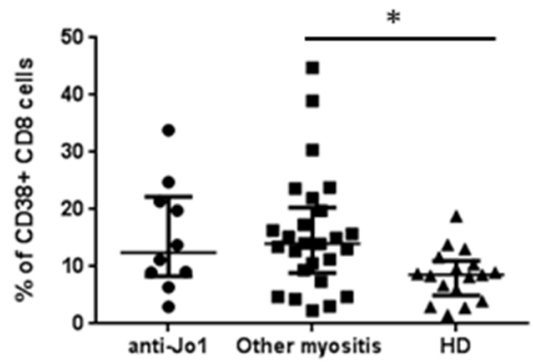

d)
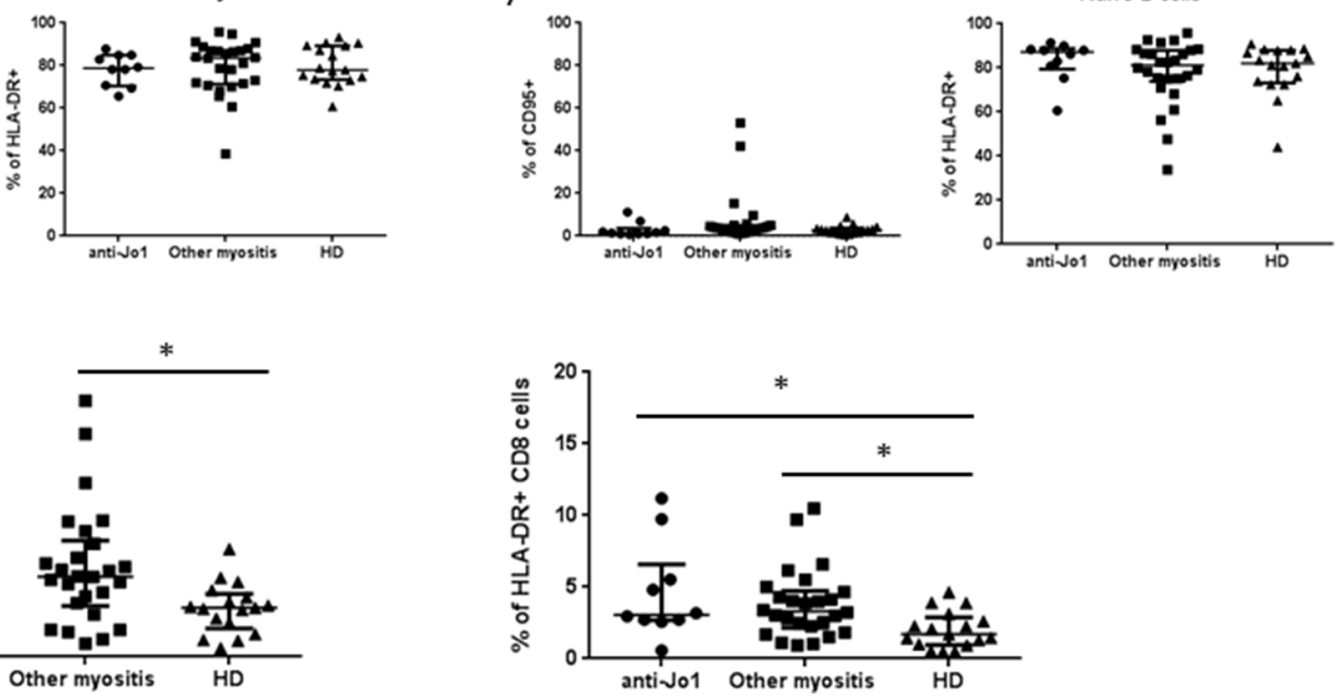

Figure 1 
Anti-Jo1 patient 1

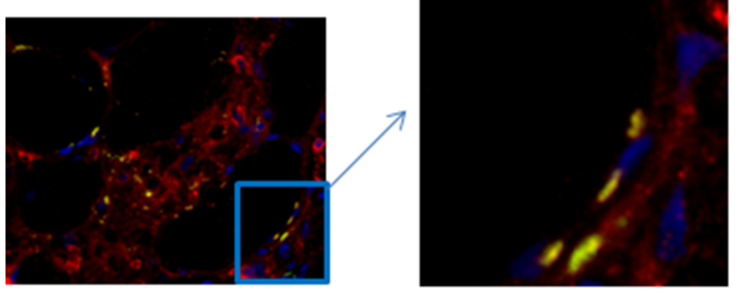

Anti-Jo1 patient 2

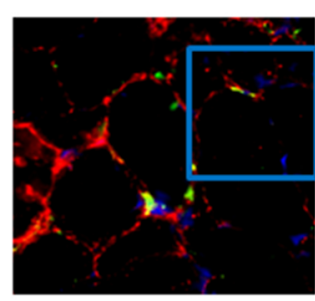

CD27 (су3)

- CD20 (AF488)

DAPI 